\title{
Immunological purification of rat precartilaginous stem cells and constuction of the immortalized cell strain
}

\author{
Shuwei Zhang ${ }^{1}$, Fengjing Guo ${ }^{1}$, Hongbo You ${ }^{1}$, Hui Liao ${ }^{1}$, Xuebi Tian $^{2}$, Dengxin Song ${ }^{1}$, Feng Gao ${ }^{2}$, Anmin Chen ${ }^{1}$ \\ ${ }^{I}$ Department of Orthopedics, ${ }^{2}$ Department of Anesthesiology, Tongji Hospital, Tongji Medical College, Huazhong University of \\ Science \& Technology, Wuhan, China
}

Precartilaginous stem cells(PCSC) are adult stem cells which control limb growth of animal and can differentiate directionally. In previous study, we found PCSC begin differentiating at the 5th passage, sufficient uniphenotype PCSC can not be harvested by primary cell culture. Therefore, we focused in this study to understand whether simian virus 40 large $T$ antigen gene (SV40Tag) can induce rat PCSC to immortalize. Immunomagnetic separation was used to isolate PCSC labeled with fibroblast growth factor receptor-3(FGFR-3). Plasmid pCMVSV40T/PUR containing SV40Tag was transfected into PCSC by liposome transfection method. One anti-puromycin cell clone was obtained, which was confirmed as FGFR-3 positive, and expanded to immortalized cell strain. Results from RT-PCR and Southern blot demonstrated that mRNA and protein of SV40Tag was highly expressed after stable transfection. The cells transfected with SV40Tag were expanded to immortalized cell strain which could maintain its characteristics for 30 passages, named immortalized precartilaginous stem cells(IPCSC). IPCSC were short fusiform or triangular cells with two or three short axons. The high proliferation capability of IPCSC was confirmed by FGFR-3, Collagen II, PCNA staining and MTT assay. Therefore, we conclude that rat precartilaginous stem cells were purifued and immortalized precartilaginous stem cell strain was established. It will provides a stable cell resource for basic research and cell transplantation therapies.

Keywords: precartilaginous stem cells, immunological purification, simian virus 40, immortalization

Cell Research (2008) 18:s153. doi: 10.1038/cr.2008.243; published online 4 August 2008

Correspondence: Shuwei Zhang

E-mail: soovycn@hotmail.com 